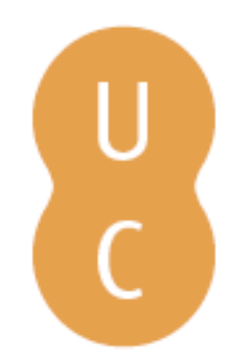

\title{
nommalina
}

\section{Upa faz a diferença: acções de sensibilização pró-saúde mental: construção do questionário de avaliação}

\author{
Autor(es): $\quad$ Campos, Luísa; Costa, Natália; Palha, Filipa \\ Publicado por: Imprensa da Universidade de Coimbra \\ URL \\ persistente: URI:http://hdl.handle.net/10316.2/35962 \\ DOI: $\quad$ DOI:http://dx.doi.org/10.14195/978-989-26-0852-5_9 \\ Accessed : $\quad$ 26-Apr-2023 13:01:50
}

A navegação consulta e descarregamento dos títulos inseridos nas Bibliotecas Digitais UC Digitalis, UC Pombalina e UC Impactum, pressupõem a aceitação plena e sem reservas dos Termos e Condições de Uso destas Bibliotecas Digitais, disponíveis em https://digitalis.uc.pt/pt-pt/termos.

Conforme exposto nos referidos Termos e Condições de Uso, o descarregamento de títulos de acesso restrito requer uma licença válida de autorização devendo o utilizador aceder ao(s) documento(s) a partir de um endereço de IP da instituição detentora da supramencionada licença.

Ao utilizador é apenas permitido o descarregamento para uso pessoal, pelo que o emprego do(s) título(s) descarregado(s) para outro fim, designadamente comercial, carece de autorização do respetivo autor ou editor da obra.

Na medida em que todas as obras da UC Digitalis se encontram protegidas pelo Código do Direito de Autor e Direitos Conexos e demais legislação aplicável, toda a cópia, parcial ou total, deste documento, nos casos em que é legalmente admitida, deverá conter ou fazer-se acompanhar por este aviso.

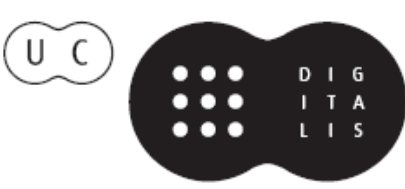





\section{UPA FAZ A DIFERENÇA - ACÇÕES DE S ENSIBILIZAÇÃO PRÓ-SAÚdE MENTAL. CONSTRUÇÃO DO QUESTIONÁRIO DE AVALIAÇÃo}

\section{Enquadramento do projecto}

A escassa informação e o estigma associado às doenças mentais é hoje reconhecido como um sério obstáculo à promoção da saúde/ saúde mental (Pinfold, Stuart, Thornicroft \& Arbolelda-Flórez, 2005; Pinfold, Toulmin, Thornicroft, Huxley, Farmer \& Graham, 2003; Schulze, Richter-Werling, Matschinger \& Angermeyer, 2003; Stuart, 2006).

As atitudes em relação à doença mental são construídas, gradualmente, sendo que têm a sua origem na infância, consolidando-se durante a idade adulta. Torna-se assim evidente a vantagem de intervir durante a juventude, altura em que as atitudes são ainda maleáveis, mais facilmente contestáveis e modificáveis (Wahl, 2002).

É neste contexto que os jovens se assumem como um grupo-alvo prioritário para o desenvolvimento de iniciativas de sensibilização sobre saúde mental.

Dados de estudos epidemiológicos realizados junto desta população demonstram que 1 em cada 5 jovens (Patel, Flisher, Hetrick \& McGorry, 2007; Stuart, 2006) irá passar pela experiência de um problema psi-

\footnotetext{
1 Professora Auxiliar da Faculdade de Educação e Psicologia da Universidade Católica. Membro da Direcção da ENCONTRAR+SE.

2 Psicóloga da ENCONTRAR+SE.

3 Docente da Faculdade de Educação e Psicologia da Universidade Católica. Presidente da Direcção da ENCONTRAR+SE
} 
cológico significativo durante a escolaridade e que muitos mais irão ter problemas psicossociais que vão interferir no seu funcionamento a diferentes níveis. Alguns trabalhos apontam mesmo para o facto de um quarto dos jovens vir a experienciar um problema de saúde mental ao longo da vida (OMS, 2008). Por outro lado, sabe-se que problemas de saúde mental se encontram fortemente associados a outras dificuldades desenvolvimentais e de saúde, tais como fraco rendimento escolar, abuso de substâncias, violência e uma saúde reprodutiva e sexual deficitária (Patel et al., 2007).

É com base no reconhecimento destes aspectos, bem como no facto da escola ser um contexto privilegiado de acesso aos jovens (Kelly, Jorm \& Wright, 2007; Wyn, Cahill, Holdsworth, Rowling \& Carson, 2000) que, durante as últimas décadas, se tem assistido ao desenvolvimento das designadas "School-based interventions".

\section{Estado da arte Internacional | Nacional}

No que diz respeito ao estado da arte internacional neste contexto, torna-se fundamental enfatizar a Austrália como um dos países pioneiros no desenvolvimento e implementação das anteriormente referidas "School-based interventions". Neste sentido, foram promovidos programas tais como o MindMatters - Understanding mental illness (Wyn et al., 2000), o Beyoundblue Schools Research Initiative - Mental Health Literacy Component (Spence, Burns, Boucher, Glover, Graetz, Kay, Patton \& Sawyer, 2005) e o Mental Illness Education (Richwood, Cavanagh, Curtis \& Sakrouge, 2004). Nos Estados Unidos foram implementados, por exemplo, o The Science of Mental Illness (Watson, Otey, Westbrook, Gardner, Lamb, Corrigan \& Fenton, 2004) e o Mental Illness Awareness Week Program (Battaglia, Coverdale \& Bushong, 1990). Por sua vez, nas escolas secundárias do Reino Unido foi implementado o Mental Health Awareness in Action program (Pinfold et al., 2003). E, finalmente, na Alemanha poderá ser salientado o projecto Crazy? So what! It's normal to be different (Richter-Werling, Matchinger \& Angermeyer, 2003). 
No que concerne a Portugal, o Grupo Aventura Social, liderado pela Professora Margarida Gaspar de Matos da Faculdade de Motricidade Humana, tem vindo a desenvolver diversas investigações no domínio da promoção da saúde (e.g., Gaspar, Pais-Ribeiro, Matos \& Leal, 2008). Paralelamente, em 2005, o Ministério da Educação criou o Grupo de Trabalho para a Educação Sexual/Educação para a Saúde no intuito de assegurar que a Educação para a Saúde seria incluída nos currículos escolares até 2007. Para tal, foram identificadas quatro áreas prioritárias: (1) o abuso de substâncias; (2) as doenças sexualmente transmissíveis; (3) a alimentação e a actividade física; e, por último, (4) a prevenção da violência e promoção do bem-estar/saúde mental.

Em síntese, e no que diz respeito ao estado da arte em Portugal, por um lado desconhece-se a existência de intervenções sistematizadas destinadas a combater o estigma associado às doenças mentais junto de jovens estudantes; e, por outro lado, considera-se que não é muito clara a forma como se pretendem tratar as questões da "doença mental" em contexto escolar. É perante este cenário que surge o projecto "UPA FAZ A DIFERENÇA - Acções de Sensibilização Pró-Saúde Mental”.

\section{Projecto UPA FAZ A DIfERENÇA}

O projecto UPA FAZ A DIFERENÇA - Acções de Sensibilização Pró-Saúde Mental, promovido pela ENCONTRAR+SE, co-finaciado pelo Alto Comissariado da Saúde, pela Fundação Calouste Gulbenkian e pela Lilly Portugal, com o apoio científico da Faculdade de Educação e Psicologia da Universidade Católica, tem como objectivo geral: contribuir para o aumento do conhecimento sobre as doenças mentais dos jovens (dos 15 aos 18 anos), no sentido de incentivar a procura precoce de ajuda e diminuir atitudes estigmatizantes e discriminatórias. Mais especificamente pretende-se: (1) avaliar os conhecimentos acerca da doença mental num grupo de 500 jovens; (2) realizar o levantamento dos mitos e crenças negativas sobre as doenças mentais num grupo de 500 jovens; (3) transmitir informações sobre os principais sinais e sintomas de alerta para 
risco de doença mental; (4) divulgar o movimento UPA - Unidos para ajudar, cujo mote é "Levanta-te contra a discriminação da doença mental", no sentido de sensibilizar para o impacto do auto/hetero-estigma; e, por fim, (5) avaliar a eficácia da iniciativa e de necessidades futuras.

Este projecto segue uma metodologia do tipo pré-pós, sendo que numa fase pré-intervenção será realizado o levantamento dos conhecimentos, mitos e crenças sobre saúde/doença mental; numa segunda fase será desenvolvida uma intervenção breve de cerca de 2 sessões, à qual se seguirá a última fase, centrada na avaliação da eficácia da intervenção.

\subsection{Estudo piloto}

Entre Setembro de 2009 e Julho de 2010, está a ser implementado um estudo piloto com o objectivo de realizar o levantamento dos conhecimentos de jovens do ensino secundário sobre os temas saúde/doença mental que permitirá, por um lado, (1) desenvolver o questionário fechado para avaliação dos conhecimentos e da eficácia da intervenção; e, por outro lado, (2) contribuir para o desenvolvimento das acções de sensibilização a realizar em meio escolar.

\subsubsection{Fase pré-piloto}

O estudo piloto integra ainda uma fase pré-piloto que seguirá uma metodologia qualitativa. Desta forma, serão realizados 6 focus groups ou grupos de discussão junto de 24 alunos do ensino secundário. Para tal serão constituídos 6 grupos de discussão, 3 numa escola pública e 3 numa escola privada.

Em cada escola serão realizados 3 grupos, um com alunos do $10 .^{\circ}$ ano, outro com alunos do $11 .^{\circ}$ e outro com alunos do $12 .^{\circ}$ ano.

Cada grupo será constituído por 8 alunos, dois de cada agrupamento (1 rapaz/1 rapariga) - 2 alunos de ciências e tecnologias, 2 alunos de ciências socioeconómicas, 2 alunos de línguas e humanísticas e 2 alunos 
de artes visuais (a selecção dos alunos, por ano lectivo e por área de ensino, será realizada de forma aleatória).

Partindo da revisão da literatura, da realização de entrevistas individuais e, posteriormente, de uma análise e discussão da equipa foi delineado um guião, que orientará a realização dos grupos de discussão, organizado em torno de oito grandes áreas: (1) definição de doença mental; (2) causas e riscos na doença mental; (3) impacto na doença mental; (4) tratamento/ prognóstico da doença mental; (5) intenções comportamentais; (6) definição de saúde mental; (7) promoção da saúde mental; e, por último, (8) levantamento de necessidades.

Estando definido o guião entramos agora na fase de realização dos grupos de reflexão, a qual decorrerá nos próximos dois meses.

\section{Referências bibliográficas}

Battaglia, J., Coverdale, J. H. \& Bushong, C. P. (1990). Evaluation of a mental illness awareness week program in public schools. American Journal of Psychiatry, 147, 324-329.

GASPAR, T., PAis-Ribeiro, J. L., MATOs, M. G. \& LEAL, I. (2008). Promoção de qualidade de vida em crianças e adolescentes. Psicologia, Saúde E Doenças, 9 (1), 55-71.

KELlY, C., JORM, A. \& WRIGHT, A. (2007). Improving mental health literacy as a stategy to facilitate early intervention for mental disorders. The Medical Journal of Australia, 187 (7), S26-S30.

ORGANIZAÇÃo MUNDIAL DE SAÚdE [OMS] (2008). Social Cohesion for Mental Well-being among adolescents. Copenhagen: WHO Regional Office for Europe.

PATEl, V., Flisher, A., HeTrick, S. \& MCGORRY, P. (2007). Mental health of young people: A global public-health challenge. The Lancet, 369, 1302-1313.

Pinfold, V., STuArt, H., ThORnicroft, G. \& ARBOlEldA-FlórEZ, J. (2005). Working with young people: The impact of mental health awareness programs in schools in the UK and Canada. World Psychiatry, 4 (1), 48-52.

Pinfold, V., Toulmin, H., Thornicroft, G., HuXley, P., FARMer, P. \& GrahAM, T. (2003). Reducing psychiatric stigma and discrimination: Evaluation of educational interventions in UK secondary schools. British Journal of Psychiatry, 182, 142-146.

RiCKWOOD, D., CAVAnAGH, S., CuRTIS, L. \& SAKROUGE, R. (2004). Educating young people about mental health and mental illness: Evaluating a school-based programme. International Journal of Mental Health Promotion, 6, 23-32.

SCHUlze, B., RiCHTER-WERling, M., MATsChinger, H. \& ANGERMEYer, M. C. (2003). Crazy? So what? Effects of a school project on student's attitudes towards people with schizophrenia. Acta Psychiatrica Scandinavica, 107, 142-50.

SPEnce, S., Burns, J., Boucher, S., Glover, S., Graetz, B., KAY, D., PATTON, G. \& SAWYER, M. (2005). The beyondblue schools research initiative: Conceptual framework and intervention. Australasian Psychiatry, 13, 159-164 
STUART, H. (2006). Reaching out to high school youth: The effectiveness of a video-based antistigma program. Canadian Journal of Psychiatry, 51, 647-653.

WAHL, O. (2002). Children's views of mental illness: A review of the literature. American Journal of Psychiatric Rehabilitation, 6 (2), 134-158.

Watson, A. C., Otey, A., Westbrook, A. L., GARDNer, A. L., LAMB, T., Corrigan, P. \& FENTON, W. (2004). Changing middle schoolers' attitudes about mental illness through education. Schizophrenia Bulletin, 30, 563-572.

WYN, J., CAHILl, H., HOLDSWORTH, R., ROWLING, L. \& CARSON, S. (2000). MindMatters, a whole-school approach promoting mental health and wellbeing. Australian and New Zealand Journal of Psychiatry, 34, 594-601. 